\title{
APPROXIMATION TO THE SOLUTION OF THE SYSTEM OF NONLINEAR STOCHASTIC DIFFERENTIAL EQUATIONS
}

\author{
VICTOR MALYUTIN \\ Institute of Mathematics, NAS of Belarus \\ 11 Surganov St. 220072 Minsk, Belarus
}

\begin{abstract}
In this paper we consider the system of nonlinear stochastic differential equations. The solution of these equations is approximated by finite series of definite integrals where every term of the series can be evaluated. To construct the approximation, the linearization technique and the technique for noncommuting matrices are used.
\end{abstract}

2000 Mathematics Subject Classification: 65C30.

Keywords: stochastic differential equations, linearization technique, chronological exponential, noncommuting matrices.

\section{Introduction}

Reaction-diffusion systems have been the subject of many experimental and theoretical studies. Such systems appear in many biological, chemical and physical processes $[5,11,12]$. Reaction-diffusion systems are described by mean-field type nonlinear equations [3,9], which do not contain the stochastic nature, and by nonlinear stochastic differential equations [8]

$$
\begin{aligned}
d x_{1}(t) & =k_{1} x_{1}(t) d t+a_{1} x_{1}(t) x_{2}(t) d t+\sigma_{1} x_{1}(t) d \omega(t) \\
d x_{2}(t) & =k_{2} x_{2}(t) d t+a_{2} x_{1}(t) x_{2}(t) d t+\sigma_{2} x_{2}(t) d \omega(t) \\
x_{1}(0) & =x_{10}, x_{2}(0)=x_{20} .
\end{aligned}
$$

where $\omega(t)$ is the Wiener process, $x_{1}(t), x_{2}(t)$ are concentrations of reactants; the coefficients of the equation $k_{i}, a_{i}, \sigma_{i}, i=1,2$ are some real or complex numbers characterizing the reaction.

There is a method for evaluating the transition probability density for the process which is the solution of one stochastic differential equation $[4,6]$. But we cannot use this method to find the solution of the system of nonlinear stochastic differential equations. It is highly nontrivial task to get an analytic solution of equation (1). Deterministic and stochastic diffusion type systems are considered by M. Arato [1]. The approach proposed in [1] gives the solution of a nonlinear stochastic differential equation as an exponential function, but the exponent contains the desired solution. Such a form of the solution gives only asymptotic behavior of the solution as $t \rightarrow \infty$. Therefore we propose an approximation for solving the nonlinear stochastic differential equations (1). The approximation is represented as a finite series of increasing multiplicity definite integrals, where every term of series can be 
evaluated. The represented approximate solution makes it possible to analyze the solution for $0 \leqslant t<\infty$.

\section{The approximate formulas to the solution of the system}

Our approximations $\tilde{x}_{1}(t), \tilde{x}_{2}(t)$ to the solution of equations (1) are as follows:

$$
\begin{aligned}
\tilde{x}_{1}= & \sum_{k=0}^{n-1} \int_{0}^{t} \cdots \int_{0}^{(k)} \frac{\partial^{k}}{\partial b_{1} \ldots \partial b_{k}} \exp \left(\int_{0}^{t} \psi_{k}(\tau)\left(f\left(\bar{s}^{(k)}, \tau\right)+1\right)\right) \\
& \times\left.\sum_{j=0}^{n} \int_{0}^{t} \cdots \int_{0}^{s_{2}} \prod_{l=1}^{j} \psi_{q k}\left(\tau_{l}\right) \exp \left(\int_{0}^{\tau_{l}} \psi_{d k}(u)\right)\right|_{b_{1}=\ldots=b_{k}=0} d s_{1} \ldots d s_{k} x_{10}^{k+1} x_{20}^{j} a_{2}^{k}, \\
\tilde{x}_{2}= & \sum_{k=0}^{n} \int_{0}^{t} \cdots \int_{0}^{t} \frac{\partial^{k}}{\partial b_{1} \ldots \partial b_{k}} \exp \left(\int_{0}^{t} \psi_{k}(\tau) f\left(\bar{s}^{(k)}, \tau\right)\right) \exp \left(\int_{0}^{s_{2}} \psi_{d k}(\tau)\right) \\
& \times \sum_{j=0}^{n-1} \int_{0}^{t} \cdots \int_{0}^{(j)} \prod_{l=1}^{\tau_{2}}\left(\psi_{q k}\left(\tau_{l}\right)-a_{1} d \tau_{l}\right) \times\left.\exp \left(\int_{0}^{\tau_{l}} \psi_{d k}(u)\right)\right|_{b_{1}=\ldots=b_{k}=0} d \bar{s}^{k} x_{10}^{k} x_{20}^{j+1} a_{2}^{k},
\end{aligned}
$$

where $f\left(\bar{s}^{(k)}, \tau\right)=\sum_{j=1}^{k+1}(k+1-j) \chi_{\left.s_{j-1}, s_{j}\right]}(\tau), s_{0}=0, s_{k+1}=t, \psi_{d k}(\tau)=\left(\phi\left(\bar{s}^{(k)}\right)+k_{2}-\right.$ $\left.\frac{1}{2} \sigma_{2}^{2}\right) d \tau+\sigma_{2} d \omega(\tau), \psi_{q k}(\tau)=a_{1}\left(f\left(\bar{s}^{(k)}\right)+1\right) d \tau, \psi_{k}(\tau)=\left(k_{1}-\frac{1}{2} \sigma_{1}^{2}\right) d \tau+\sigma_{1} d \omega(\tau), k \geqslant 0$, $\phi\left(\bar{s}^{(k)}, \tau\right)=\sum_{j=1}^{k} b_{k} \delta\left(\tau-s_{j}\right)$.

The construction of the approximation or proof of equalities (2) will be divided into several steps. In first step we reduce the nonlinear equation to a linear one and introduce the matrices $D, Q, d, q$; in the second step, we remove the matrix $D$; in the third step, we remove the matrix $d$; the fourth step is to remove the matrices $Q, q$.

\subsection{Reduction of the nonlinear equation to a linear one}

Using the linearization technique of [7], in order to find solutions $x_{1}, x_{2}$ we introduce $y_{i j}(t)=$ $x_{1}^{i}(t) x_{2}^{j}(t), i, j=0,1,2, \ldots$ Then using the Ito formula [4] we get the expression for $d y_{i j}(t)$ :

$$
\begin{aligned}
d y_{i j}(t)= & \left(i k_{1}+j k_{2}\right) y_{i j}(t) d t+\left(i a_{1} y_{i j+1}(t)+j a_{2} y_{i+1 j}(t)\right) d t \\
& +\left(i \sigma_{1}+j \sigma_{2}\right) y_{i j}(t) d \omega(t)+i j \sigma_{1} \sigma_{2} y_{i j}(t) d t \\
& +\frac{1}{2}\left(i(i-1) \sigma_{1}^{2}+j(j-1) \sigma_{2}^{2}\right) y_{i j}(t) d t
\end{aligned}
$$

If $y_{i j}(t), i, j=0,1,2, \ldots$ are the solutions of system $(3)$, then $x_{1}(t)=y_{10}(t), x_{2}(t)=y_{01}(t)$ are the solutions of system (1).

Let us consider $\tilde{x}_{1}(t)=y_{10}(t), \tilde{x}_{2}(t)=y_{01}(t)$ - the solutions of the system of equation $(3)$, where $i, j \leqslant n$, as approximation of $x_{1}(t), x_{2}(t)$ from system $(1)$. The convergence $\tilde{x}_{1}(t), \tilde{x}_{2}(t)$ to $x_{1}(t), x_{2}(t)$ will be discussed in 2.5 .

Let $\vec{y}$ be $\left(\overrightarrow{y_{0}}, \overrightarrow{y_{1}}, \ldots, \overrightarrow{y_{n}}\right), \overrightarrow{y_{i}}=\left(y_{i 0}, y_{i 1}, \ldots, y_{i n}\right), i=0,1, \ldots, n, \vec{y}(0)=\left(\overrightarrow{x_{20}}, x_{10} \overrightarrow{x_{20}}, \ldots\right.$, $\left.x_{10}^{n} \overrightarrow{x_{20}}\right), \overrightarrow{x_{20}}=\left(1, x_{20}, x_{20}^{2}, \ldots, x_{20}^{n}\right)$. To write equation (3) in matrix form, let us introduce 
the matrices

$$
d=\left(\begin{array}{cccccc}
0 & 0 & 0 & \ldots & 0 & 0 \\
0 & 1 & 0 & \ldots & 0 & 0 \\
0 & 0 & 2 & \ldots & 0 & 0 \\
\vdots & \vdots & \vdots & \ddots & \vdots & \vdots \\
0 & 0 & 0 & \ldots & 0 & n
\end{array}\right), \quad q=\left(\begin{array}{cccccc}
0 & 1 & 0 & \ldots & 0 & 0 \\
0 & 0 & 1 & \ldots & 0 & 0 \\
\vdots & \vdots & \vdots & \ddots & \vdots & \vdots \\
0 & 0 & 0 & \ldots & 0 & 1 \\
0 & 0 & 0 & \ldots & 0 & 0
\end{array}\right)
$$

which have dimensions $(n+1) \times(n+1)$ and satisfy the commutative rules $q d-d q=q$, we also introduce the matrices

$$
D=\left(\begin{array}{cccccc}
0 e & 0 & 0 & \ldots & 0 & 0 \\
0 & 1 e & 0 & \ldots & 0 & 0 \\
0 & 0 & 2 e & \ldots & 0 & 0 \\
\vdots & \vdots & \vdots & \ddots & \vdots & \vdots \\
0 & 0 & 0 & \ldots & 0 & n e
\end{array}\right), \quad Q=\left(\begin{array}{cccccc}
0 & e & 0 & \ldots & 0 & 0 \\
0 & 0 & e & \ldots & 0 & 0 \\
\vdots & \vdots & \vdots & \ddots & \vdots & \vdots \\
0 & 0 & 0 & \ldots & 0 & e \\
0 & 0 & 0 & \ldots & 0 & 0
\end{array}\right)
$$

which have dimensions $(n+1)^{2} \times(n+1)^{2}$ and satisfy the commutative rules $Q D-D Q=Q$, $e$ is the $(n+1) \times(n+1)$ identical matrix, $E$ is the $(n+1)^{2} \times(n+1)^{2}$ identical matrix.

Now we can write equation (3) as a matrix linear stochastic differential equation

$$
\begin{aligned}
d \vec{y}(t)= & \left(D \sigma_{1}+d E \sigma_{2}\right) \vec{y}(t) d \omega(t)+k_{1} D+k_{2} d E+a_{1} D q+a_{2} d Q \\
& \left.+d D \sigma_{1} \sigma_{2}+\frac{1}{2} D(D-E) \sigma_{1}^{2}+\frac{1}{2} d E(d-e) \sigma_{2}^{2}\right) \vec{y}(t) d t
\end{aligned}
$$

Using the definition of the chronological exponential or $T \exp$ [10], we get

$$
\vec{y}(t)=T \exp \left\{\int_{0}^{t}\left(H(D, d)+a_{1} D q+a_{2} d Q\right)\right\} \vec{y}(0),
$$

where $H(D, d)=\left(k_{1} D+k_{2} d E-\frac{1}{2} D \sigma_{1}^{2}-\frac{1}{2} d E \sigma_{2}^{2}\right) d s+\left(D \sigma_{1}+d E \sigma_{2}\right) d \omega(s)$.

\subsection{Removal of matrix $D$}

Using the equality

$$
\begin{aligned}
& T \exp \left\{\int_{0}^{t}\left(H(D, d)+a_{1} D q+a_{2} d Q\right)\right\} \\
& =T \exp \left\{\int_{0}^{t}\left(H(D, d)+a_{1} D q\right)\right\}+\int_{0}^{t} T \exp \left\{\int_{s_{1}}^{t}\left(H(D, d)+a_{1} D q\right)\right\} a_{2} d Q \\
& \quad \times T \exp \left\{\int_{0}^{s_{1}}\left(H(D, d)+a_{1} D q+a_{2} d Q\right)\right\} d s_{1}
\end{aligned}
$$


we can write $T \exp$ as the series

$$
\begin{aligned}
T & =\left\{\int_{0}^{t}\left(H(D, d)+a_{1} D q+a_{2} d Q\right)\right\} \\
= & T \exp \left\{\int_{0}^{t}\left(H(D, d)+a_{1} D q\right)\right\}+\int_{0}^{t} T \exp \left\{\int_{s_{1}}^{t}\left(H(D, d)+a_{1} D q\right)\right\} a_{2} d Q \\
& \times T \exp \left\{\int_{0}^{s_{1}}\left(H(D, d)+a_{1} D q\right)\right\} d s_{1}+\ldots \\
& +\int_{0}^{t}(k) \int_{0}^{s_{2}} T \exp \left\{\int_{s_{k}}^{t}\left(H(D, d)+a_{1} D q\right)\right\} a_{2} d Q \\
& \times T \exp \left\{\int_{s_{k-1}}^{s_{k}}\left(H(D, d)+a_{1} D q\right)\right\} a_{2} d Q \times \cdots \times T \exp \left\{\int_{s_{1}}^{s_{2}}\left(H(D, d)+a_{1} D q\right)\right\} a_{2} d Q \\
& \times T \exp \left\{\int_{0}^{s_{1}}\left(H(D, d)+a_{1} D q\right)\right\} d s_{1} \ldots d s_{k}+\ldots
\end{aligned}
$$

We consider separately the evaluation of $\tilde{x}_{1}$ and $\tilde{x}_{2}$. For that we need to remove the matrices $D, d, Q, q$. Let us start with the removal of the matrix $D$ and use a technique analogous to the quantum mechanics technique to evaluate the vacuum mean of the operator [2]. Here the creating operators are relocated to the left, the annihilator operators are relocated to the right, and the action of the annihilator operator on vacuum is zero.

To evaluate $\tilde{x}_{1}$, we need to know $(n+2)$-th component of the vector $T \exp \left\{\int_{0}^{t}(H(D, d)\right.$ $\left.\left.+a_{1} D q+a_{2} d Q\right)\right\} \vec{y}(0)$. Since after the action of the matrix $D-E$ on any vector the $(n+2)$-th component of the resulting vector is zero, we relocate the matrices $D$ in the expression for $T \exp$ to the left and relocate the matrices $Q$ to the right. After relocating the matrices $D$ and $Q$ and taking into account that $Q f(D)=f(D+E) Q$ and for any vector $\vec{z}$ the $(n+2)$-th component of $((D-E) \vec{z})_{n+2}$ is zero, we get the following expression for $T \exp$ :

$$
\begin{aligned}
T & \exp \left\{\int_{0}^{t}\left(H(D, d)+a_{1} D q+a_{2} d Q\right)\right\} \\
= & T \exp \left\{\int_{0}^{t}\left(H(1, d)+a_{1} q E\right)\right\}+\sum_{k=1}^{\infty} \int_{0}^{t}(k) \int_{0}^{s_{2}} T \exp \left\{\int_{s_{k}}^{t}\left(H(1, d)+a_{1} q E\right)\right\} a_{2} E d \\
& \times T \exp \left\{\int_{s_{k-1}}^{s_{k}}\left(H(2, d)+2 a_{1} q E\right)\right\} a_{2} E d \times \cdots \times T \exp \left\{\int_{s_{1}}^{s_{2}}\left(H(k, d)+k a_{1} q E\right)\right\} \\
& \times a_{2} E d \times T \exp \left\{\int_{0}^{s_{1}}\left(H(k+1, d)+(k+1) a_{1} q E\right)\right\} d s_{1} \ldots d s_{k} Q^{k} .
\end{aligned}
$$


This expression does not contain the matrix $D$, and we have numbers instead of $D$. Thus, the process of removal of the matrix $D$ from the expression for $T \exp$ is finished.

To evaluate $\tilde{x}_{2}$, we need 2 -th component of the vector $\vec{y}$. Since after the action of the matrix $D$ on any vector the 2 -th component of the resulting vector is zero, we relocate the matrices $D$ in expression (6) for $T \exp$ to the left and relocate the matrices $Q$ to the right. After relocating the matrices $D$ and $Q$ and taking into account that $Q f(D)=f(D+E) Q$ and for any vector $\vec{z}$ the 2-th component of $(D \vec{z})_{2}$ is zero, we get the following expression for $T$ exp:

$$
\begin{aligned}
T & \exp \left\{\int_{0}^{t}\left(H(D, d)+a_{1} D q+a_{2} d Q\right)\right\} \\
= & T \exp \left\{\int_{0}^{t}\left(H(0, d)+0 a_{1} q E\right)\right\}+\sum_{k=1}^{\infty} \int_{0}^{t} \cdots \int_{0}^{s_{2}} T \exp \left\{\int_{s_{k}}^{t}\left(H(0, d)+0 a_{1} q E\right)\right\} a_{2} E d \\
& \times T \exp \left\{\int_{s_{k-1}}^{s_{k}}\left(H(1, d)+1 a_{1} q E\right)\right\} a_{2} E d \times \cdots \\
& \times T \exp \left\{\int_{s_{1}}^{s_{2}}\left(H(k-1, d)+(k-1) a_{1} q E\right)\right\} \\
& \times a_{2} E d \times T \exp \left\{\int_{0}^{s_{1}}\left(H(k, d)+k a_{1} q E\right)\right\} d s_{1} \ldots d s_{k} Q^{k} .
\end{aligned}
$$

\subsection{Removal of matrix $d$}

Represent the product of $T$ exp under the integral sign in (7) as single $T$ exp. In addition, in the exponent of $T$ exp we group together the terms containing the matrix $d$ and the terms containing the matrix $q$. Let the coefficient before the matrix $d$ be $\psi_{d}$, the coefficient before the matrix $q$ be $\psi_{q}$, and the term without matrices $d$ and $q$ be $\psi$. Then

$$
\begin{aligned}
& T \exp \left\{\int_{s_{k}}^{t}\left(H(1, d)+a_{1} q E\right)\right\} E d \times T \exp \left\{\int_{s_{k-1}}^{s_{k}}\left(H(2, d)+2 a_{1} q E\right)\right\} E d \times \cdots \\
& \times T \exp \left\{\int_{s_{1}}^{s_{2}}\left(H(k, d)+k a_{1} q E\right)\right\} E d \times T \exp \left\{\int_{0}^{s_{1}}\left(H(k+1, d)+(k+1) a_{1} q E\right)\right\} \\
& =\left.\frac{\partial^{k}}{\partial b_{1} \ldots \partial b_{k}} T \exp \left\{\int_{0}^{t}\left(\psi_{d k} d+\psi_{q k} q+\left(f\left(\bar{s}^{(k)}\right)+1\right) \psi_{k}\right)\right\}\right|_{b_{1}=\ldots=b_{k}=0}
\end{aligned}
$$

To represent $T \exp \left\{\int_{0}^{t}\left(\psi_{d k}(\tau) d+\psi_{q k}(\tau) q+\psi_{k}(\tau)\left(f\left(\bar{s}^{(k)}\right)+1\right)\right)\right\}$ in the form of exponen- 
tial without the chronological ordered sign, write $T \exp$ in the following form:

$$
\begin{aligned}
& T \exp \left\{\int_{0}^{t}\left(\psi_{d k}(\tau) d+\psi_{q k}(\tau) q+\psi_{k}(\tau)\left(f\left(\bar{s}^{(k)}\right)+1\right)\right)\right\}=\exp \left(\int_{0}^{t} \psi_{k}(\tau)\left(f\left(\bar{s}^{(k)}\right)+1\right)\right) \\
& \quad \times\left(\exp \left\{\int_{0}^{t} d \psi_{d k}(\tau)\right\}+\sum_{j=1}^{\infty} \int_{0}^{t} \cdots \int_{0}^{(j)} \operatorname{sexp}\left(\int_{s_{j}}^{t} d \psi_{d k}(\tau)\right) q \psi_{q k}\left(s_{j}\right)\right. \\
& \times \exp \left\{\int_{s_{j-1}}^{s_{j}} d \psi_{d k}(\tau)\right\} q \psi_{q k}\left(s_{j-1}\right) \times \cdots \\
& \left.\quad \times \exp \left\{\int_{s_{1}}^{s_{2}} d \psi_{d k}(\tau)\right\} q \psi_{q k}\left(s_{1}\right) \times \exp \left\{\int_{0}^{s_{1}} d \psi_{d k}(\tau)\right\} d s_{1} \ldots d s_{j}\right) .
\end{aligned}
$$

Because after the action of the matrix $d$ on any vector the $(n+2)$-th component of the resulting vector is zero, we relocate the matrices $d$ to the left and the matrices $q$ to the right in the expression for $T$ exp. Then taking into account that $q f(d)=f(d+e) q$ and for any vector $\vec{z}$ the $(n+2)$-th component of $(d \vec{z})_{n+2}$ is zero, we obtain

$$
\begin{aligned}
& T \exp \left\{\int_{0}^{t}\left(\psi_{d k}(\tau) d+\psi_{q k}(\tau) q+\psi_{k}(\tau)\left(f\left(\bar{s}^{(k)}\right)+1\right)\right)\right\}=\exp \left(\int_{0}^{t} \psi_{k}(\tau)\left(f\left(\bar{s}^{(k)}\right)+1\right)\right) \\
& \quad \times\left(1+\sum_{j=1}^{\infty} \int_{0}^{t} \stackrel{(j)}{s_{2}} \exp \left(\int_{0}^{t} 0 \psi_{d k}(\tau)\right) \psi_{q k}\left(s_{j}\right) \times \exp \left\{\int_{s_{j-1}}^{s_{j}} 1 \psi_{d k}(\tau)\right\} \psi_{q k}\left(s_{j-1}\right) \times \cdots\right. \\
& \left.\quad \times \exp \left\{\int_{s_{1}}^{s_{2}}(j-1) d \psi_{d k}(\tau)\right\} \psi_{q k}\left(s_{1}\right) \times \exp \left\{\int_{0}^{s_{1}} j \psi_{d k}(\tau)\right\} d s_{1} \ldots d s_{j} q^{j}\right) .
\end{aligned}
$$

Let $P_{k}(t)$ be $T \exp \left\{\int_{0}^{t}\left(\psi_{d k}(\tau) d+\psi_{q k}(\tau) q\right)\right\}$ and evaluate the differential of the function $P_{k}(t)$.

$$
d P_{k}(t)=\psi_{q k}(t) q \exp \left(\int_{0}^{t} \psi_{d k}(\tau)\right) P_{k}(t), P_{k}(0)=1 .
$$

Solving this equation, one gets the expression for $P_{k}(t)$ and for $T \exp$

$$
\begin{aligned}
T & \exp \left\{\int_{0}^{t}\left(\psi_{d k}(\tau) d+\psi_{q k}(\tau) q+\psi_{k}(\tau)\left(f\left(\bar{s}^{(k)}\right)+1\right)\right)\right\} \\
& =\exp \left(\int_{0}^{t} \psi_{k}(\tau)\left(f\left(\bar{s}^{(k)}\right)+1\right)\right) \exp \left(q \int_{0}^{t} \psi_{q k}(\tau) \exp \left(\int_{0}^{\tau} \psi_{d k}(u)\right)\right) .
\end{aligned}
$$


From equalities (5), (7)-(9) one infers that the solution $\tilde{x}_{1}$ is the $(n+2)$-th component of the vector

$$
\begin{aligned}
& \left(\exp \left(\int_{0}^{t} \psi_{0}(\tau)\right) \exp \left(q \int_{0}^{t} \psi_{q 0}(\tau) \exp \left(\int_{0}^{\tau} \psi_{d 0}(s)\right)\right)\right. \\
& \quad+\sum_{k=1}^{\infty} \int_{0}^{t} \stackrel{(k)}{s_{2}} \frac{\partial^{k}}{\partial b_{1} \ldots \partial b_{k}} \exp \left(\int_{0}^{t} \psi_{k}\left(f\left(\bar{s}^{(k)}\right)+1\right) d \tau\right) \\
& \left.\quad \times\left.\exp \left(q \int_{0}^{t} \psi_{q k}(\tau) \exp \left(\int_{0}^{\tau} \psi_{d k}(u)\right)\right)\right|_{b_{1}=\ldots=b_{k}=0} d s_{1} \ldots d s_{k} a_{2}^{k} Q^{k}\right) \vec{y}(0) .
\end{aligned}
$$

Let us write the product of $T$ exp under the integral sign in expression $\left(7^{\prime}\right)$ as single $T \exp$. In addition, in the exponent of $T \exp$ we group together the terms containing the matrix $d$ and the terms containing the matrix $q$. Then

$$
\begin{aligned}
& T \exp \left\{\int_{s_{k}}^{t}\left(H(0, d)+0 a_{1} q E\right)\right\} E d \times T \exp \left\{\int_{s_{k-1}}^{s_{k}}\left(H(1, d)+1 a_{1} q E\right)\right\} \times E d \times \cdots \\
& \times T \exp \left\{\int_{s_{1}}^{s_{2}}\left(H((k-1), d)+(k-1) a_{1} q E\right)\right\} E d \times T \exp \left\{\int_{0}^{s_{1}}\left(H(k, d)+k a_{1} q E\right)\right\} \\
& =\left.\frac{\partial^{k}}{\partial b_{1} \ldots \partial b_{k}} T \exp \left\{\int_{0}^{t}\left(\psi_{d k}(\tau) d+\left(\psi_{q k}(\tau)-a_{1} d \tau\right) q+\psi_{k}(\tau) f\left(\bar{s}^{(k)}\right)\right)\right\}\right|_{b_{1}=\ldots=b_{k}=0}
\end{aligned}
$$

Because after the action of the matrix $d-1$ on any vector the 2 -th component of the resulting vector is zero, we will have

$$
\begin{aligned}
T & \exp \left\{\int_{0}^{t}\left(\psi_{d k}(\tau) d+\left(\psi_{q k}(\tau)-a_{1} d \tau\right) q+\psi_{k}(\tau) f\left(\bar{s}^{(k)}\right)\right)\right\} \\
= & \exp \left(\int_{0}^{t} \psi_{k}(\tau) f\left(\bar{s}^{(k)}\right)\right)\left(\exp \left(\int_{0}^{t} \psi_{d k}(\tau)\right)\right. \\
& +\sum_{j=1}^{\infty} \int_{0}^{t} \cdots \int_{0}^{(j)} \exp \left(\int_{s_{j}}^{s_{2}} 1 \psi_{d k}(\tau)\right)\left(\psi_{q k}\left(s_{j}\right)-a_{1}\right) \\
& \times \exp \left\{\int_{s_{j-1}}^{t} 2 \psi_{d k}(\tau)\right\}\left(\psi_{q k}\left(s_{j-1}\right)-a_{1}\right) \times \cdots \times \exp \left\{\int_{s_{1}}^{s_{2}} j \psi_{d k}(\tau)\right\} \\
& \left.\times\left(\psi_{q k}\left(s_{1}\right)-a_{1}\right) \times \exp \left\{\int_{0}^{s_{1}}(j+1) \psi_{d k}(\tau)\right\} d s_{1} \ldots d s_{j} q^{j}\right) .
\end{aligned}
$$


Let $R_{k}(t)$ be

$$
T \exp \left\{\int_{0}^{t}\left(\psi_{d k}(\tau) d+\left(\psi_{q k}(\tau)-a_{1} d \tau\right) q\right)\right\} \times \exp \left\{-\int_{0}^{t} \psi_{d k}(\tau)\right\}
$$

and evaluate the differential of the function $R_{k}(t)$.

$$
d R_{k}(t)=\psi_{q k}(t) q \exp \left(\int_{0}^{t} \psi_{d k}(\tau)\right) R_{k}(t), R_{k}(0)=1
$$

Solving this equation, we obtain the expression for $R_{k}(t)$ and for $T \exp$

$$
\begin{aligned}
T & \exp \left\{\int_{0}^{t}\left(\psi_{d k}(\tau) d+\left(\psi_{q k}(\tau)-a_{1} d \tau\right) q+\psi_{k}(\tau) f\left(\bar{s}^{(k)}\right)\right)\right\} \\
= & \exp \left(\int_{0}^{t} \psi_{k}(\tau) f\left(\bar{s}^{(k)}\right)\right) \exp \left(\int_{0}^{t} \psi_{d k}(\tau)\right) \\
& \times \exp \left(q \int_{0}^{t}\left(\psi_{q k}(\tau)-a_{1} d \tau\right) \exp \left(\int_{0}^{\tau} \psi_{d k}(u)\right)\right) .
\end{aligned}
$$

From $(5),\left(7^{\prime}\right)-\left(9^{\prime}\right)$ one infers that the solution $\tilde{x}_{2}$ is 2 -th component of the vector

$$
\begin{aligned}
& \left(\exp \left(\int_{0}^{t} \psi_{d 0}(\tau)\right)+\sum_{k=1}^{\infty} \int_{0}^{t} \underline{(k)} \int_{0}^{s_{2}} \frac{\partial^{k}}{\partial b_{1} \ldots \partial b_{k}} \exp \left(\int_{0}^{t} \psi_{k} f\left(\bar{s}^{(k)}\right) d \tau\right)\right. \\
& \times \exp \left(\int_{0}^{t} \psi_{d k}(\tau)\right) \exp \left[q \int_{0}^{t}\left(\psi_{q k}(\tau)-a_{1} d \tau\right)\right. \\
& \left.\left.\times \exp \left(\int_{0}^{\tau} \psi_{d k}(u)\right)\right]\left.\right|_{b_{1}=\ldots=b_{k}=0} d s_{1} \ldots d s_{k} a_{2}^{k} Q^{k}\right) \vec{y}(0) .
\end{aligned}
$$

\subsection{Removal of matrices $Q, q$}

This is the last stage in the construction of the approximation. At this stage the matrices $Q, q$ are removed. Thus, the removal of all introduced matrices is finished. Expanding the exponential containing the matrix $q$, with respect to the degree of $q$ and using the equality

$$
\left(q^{j} Q^{k} \vec{y}(0)\right)_{n+2}=x_{10}^{k+1} x_{20}^{j}, k \leqslant n-1, j \leqslant n,
$$

one can write

$$
\begin{aligned}
\tilde{x}_{1}= & \sum_{k=0}^{n-1} \int_{0}^{t} \cdots \int_{0}^{s_{2}} \frac{\partial^{k}}{\partial b_{1} \ldots \partial b_{k}} \exp \left(\int_{0}^{t} \psi_{k}(\tau)\left(f\left(\bar{s}^{(k)}\right)+1\right)\right) \\
& \times\left.\sum_{j=0}^{n} \int_{0}^{t} \cdots \int_{0}^{(j)} \prod_{l=1}^{\tau_{2}} \psi_{q k}\left(\tau_{l}\right) \exp \left(\int_{0}^{\tau_{l}} \psi_{d k}(u)\right)\right|_{b_{1}=\ldots=b_{k}=0} d s_{1} \ldots d s_{k} x_{10}^{k+1} x_{20}^{j} a_{2}^{k} .
\end{aligned}
$$


Returning from $\tilde{x}_{1}$ to $\tilde{x}_{2}$, let us expand the exponential containing the matrix $q$ with respect to the degree of $q$ and using the equality

$$
\left(q^{j} Q^{k} \vec{y}(0)\right)_{2}=x_{10}^{k} x_{20}^{j+1}, k \leqslant n, j \leqslant n-1,
$$

one gets

$$
\begin{aligned}
\tilde{x}_{2}= & \sum_{k=0}^{n} \int_{0}^{t} \cdots \int_{0}^{s_{2}} \frac{\partial^{k}}{\partial b_{1} \ldots \partial b_{k}} \exp \left(\int_{0}^{t} \psi_{k}(\tau) f\left(\bar{s}^{(k)}\right)\right) \\
& \times \exp \left(\int_{0}^{t} \psi_{d k}(\tau)\right) \sum_{j=0}^{n-1} \int_{0}^{t} \cdots \int_{0}^{\tau_{2}} \prod_{l=1}^{j}\left(\psi_{q k}\left(\tau_{l}\right)-a_{1} d \tau_{l}\right) \\
& \times\left.\exp \left(\int_{0}^{\tau_{l}} \psi_{d k}(u)\right)\right|_{b_{1}=\ldots=b_{k}=0} d \bar{s}^{k} x_{10}^{k} x_{20}^{j+1} a_{2}^{k} .
\end{aligned}
$$

Thus, equalities (2) are proved and the construction of the approximation is finished.

\subsection{Convergence of the approximation}

Now let us discuss the convergence of solutions of the truncated system $\tilde{x}_{1}(t), \tilde{x}_{2}(t)$ as the dimension of the truncated system $n$ goes to infinity. To investigate the convergence of $\tilde{x}_{1}(t)$, consider the $k$-th term of $\tilde{x}_{1}(t)$ in the form

$$
\begin{aligned}
\tilde{x}_{1, k}= & \int_{0}^{t}(k) \int_{0}^{s_{2}} \frac{\partial^{k}}{\partial b_{1} \ldots \partial b_{k}} \exp \left(\int_{0}^{t} \psi_{k}(\tau)\left(f\left(\bar{s}^{(k)}, \tau\right)+1\right)\right) \\
& \times\left.\exp \left[x_{20} \int_{0}^{t} \psi_{q k}(\tau) \exp \left(\int_{0}^{\tau} \psi_{d k}(u)\right)\right]\right|_{b_{1}=\ldots=b_{k}=0} d s_{1} \ldots d s_{k} x_{10}^{k+1} a_{2}^{k} .
\end{aligned}
$$

Evaluating the derivatives, we get

$$
\begin{aligned}
\left|\tilde{x}_{1, k}\right| \leqslant & \int_{0}^{t} \cdots \int_{0}^{(k)} \int_{0}^{s_{2}} \exp \left(\int_{0}^{t} \psi_{k}(\tau)\left(f\left(\bar{s}^{(k)}, \tau\right)+1\right)\right) \\
& \times\left(\mid x_{20} \int_{0}^{t} \psi_{q k}(\tau) \exp \left(\int_{0}^{\tau}\left(\psi_{d k}(u)-\phi\left(\bar{s}^{(k)}, u\right)\right)\right)\right) \\
& \times \exp \left[x_{20} \int_{0}^{t} \psi_{q k}(\tau) \exp \left(\int_{0}^{\tau}\left(\psi_{d k}(u)-\phi\left(\bar{s}^{(k)}, u\right)\right)\right)\right] d s_{1} \ldots d s_{k}\left|x_{10}^{k+1} a_{2}^{k}\right|
\end{aligned}
$$

where $(c)_{k}=c \cdot(c+1) \cdot \ldots \cdot(c+k-1)$. 
Estimating the expression $\left(\left|x_{20} \int_{0}^{t} \psi_{q k}(\tau) \exp \left(\int_{0}^{\tau}\left(\psi_{d k}(u)-\phi\left(\bar{s}^{(k)}, u\right)\right)\right)\right|\right)_{k}$, one gets

$$
\begin{aligned}
\left|\tilde{x}_{1, k}\right| \leqslant & \int_{0}^{t} \cdots \int_{0}^{(k)} \int_{0}^{s_{2}} \exp \left(\int_{0}^{t} \psi_{k}(\tau)\left(f\left(\bar{s}^{(k)}, \tau\right)+1\right)\right) 2^{k-1} k ! \\
& \times \exp \left((1-\ln (2))\left[x_{20} \int_{0}^{t} \psi_{q k}(\tau) \exp \left(\int_{0}^{\tau}\left(\psi_{d k}(u)-\phi\left(\bar{s}^{(k)}, u\right)\right)\right)\right]\right) d s_{1} \ldots d s_{k}\left|x_{10}^{k+1} a_{2}^{k}\right| .
\end{aligned}
$$

If $\sigma_{1}, \sigma_{2}$ are imaginary and $x_{20} a_{1}<0$, using the estimation for $\psi_{k}(\tau)$ and $\psi_{d k}(u)$, we have

$$
\begin{aligned}
\left|\tilde{x}_{1, k}\right| \leqslant & \left|x_{10}^{k+1} a_{2}^{k}\right| \int_{0}^{t} \cdots \int_{0}^{s_{2}} \exp \left(\left(k_{1}-\frac{\sigma_{1}^{2}}{2}\right)\left(s_{1}+\ldots+s_{k}+t\right)\right) 2^{k-1} k ! \\
& \times \exp \left((1-\ln (2)) x_{20} a_{1}\left(s_{1}+\ldots+s_{k}+t\right) \min \left\{1, \exp \left(\left(k_{2}-\frac{\sigma_{2}^{2}}{2}\right) t\right)\right\}\right) d s_{1} \ldots d s_{k} .
\end{aligned}
$$

For $C=\min \left\{1, \exp \left(\left(k_{2}-\frac{\sigma_{2}^{2}}{2}\right) t\right)\right\}(1-\ln 2) x_{20} a_{1}+k_{1}-\frac{\sigma_{1}^{2}}{2}<0$, evaluating the integrals, we get

$$
\left|\tilde{x}_{1, k}\right| \leqslant\left|x_{10}^{k+1} a_{2}^{k}\right| 2^{k-1} k ! \frac{\exp (C t)}{k !|C|^{k}} .
$$

If $\delta=\left|\frac{2 x_{10} a_{2}}{C}\right|<1 \tilde{x}_{1}(t)$ has a limiting value as the dimension of the truncated system $n$ tends to infinity and we suppose that this limiting value is the solution of infinite system. The estimate for the error of the transition from the infinite to the truncated system is $\frac{\left|x_{10}\right|}{2} \exp (C t) \sum_{j=n}^{\infty} \delta^{j} \leqslant \frac{\left|x_{10}\right|}{2} \exp (C t) \frac{\delta^{n}}{1-\delta}$.

Analyzing the convergence of $\tilde{x}_{2}(t)$, consider the $k$-th term of $\tilde{x}_{2}(t)$ in the form

$$
\begin{aligned}
\tilde{x}_{2 k}= & \int_{0}^{t} \cdots \int_{0}^{s_{2}} \frac{\partial^{k}}{\partial b_{1} \ldots \partial b_{k}} \exp \left(\int_{0}^{t} \psi_{k}(\tau) f\left(\bar{s}^{(k)}, \tau\right)\right) \\
& \times \exp \left(\int_{0}^{t} \psi_{d k}(\tau)\right) \exp \left(x_{20} \int_{0}^{t}\left(\psi_{q k}(\tau)-a_{1} d \tau\right)\right. \\
& \left.\times \exp \left(\int_{0}^{\tau} \psi_{d k}(u)\right)\right)\left.\right|_{b_{1}=\ldots=b_{k}=0} d \bar{s}^{k} x_{10}^{k} x_{20} a_{2}^{k} .
\end{aligned}
$$

After evaluating the derivatives we will have

$$
\begin{aligned}
\left|\tilde{x}_{2 k}\right| \leqslant & \int_{0}^{t} \cdots \int_{0}^{s_{2}} \exp \left(\int_{0}^{t} \psi_{k}(\tau) f\left(\bar{s}^{(k)}, \tau\right)\right) \\
& \times\left(\mid x_{20} \int_{0}^{t}\left(\psi_{q k}(\tau)-a_{1} d \tau\right) \times \exp \left(\int_{0}^{\tau}\left(\psi_{d k}(u)-\phi\left(\bar{s}^{(k)}, u\right)\right)\right)\right) \\
& \times \exp \left(x_{20} \int_{0}^{t}\left(\psi_{q k}(\tau)-a_{1} d \tau\right) \times \exp \left(\int_{0}^{\tau}\left(\psi_{d k}(u)-\phi\left(\bar{s}^{(k)}, u\right)\right)\right)\right) d \bar{s}^{k}\left|x_{10}^{k} x_{20} a_{2}^{k}\right| .
\end{aligned}
$$


Substituting the estimate of $\left(\left|x_{20} \int_{0}^{t}\left(\psi_{q k}(\tau)-a_{1} d \tau\right) \times \exp \left(\int_{0}^{\tau}\left(\psi_{d k}(u)-\phi\left(\bar{s}^{(k)}, u\right)\right)\right)\right|\right)_{k+1}$ we get

$$
\begin{aligned}
\left|\tilde{x}_{2 k}\right| \leqslant & \int_{0}^{t} \stackrel{(k)}{\cdots} \int_{0}^{s_{2}} \exp \left(\int_{0}^{t} \psi_{k}(\tau) f\left(\bar{s}^{(k)}, \tau\right)\right) \\
& \times 2^{k}(k+1) ! \exp \left(x_{20}(1-\ln (2)) \int_{0}^{t}\left(\psi_{q k}(\tau)-a_{1} d \tau\right)\right. \\
& \left.\times \exp \left(\int_{0}^{\tau}\left(\psi_{d k}(u)-\phi\left(\bar{s}^{(k)}, u\right)\right)\right)\right) d \bar{s}^{k}\left|x_{10}^{k} x_{20} a_{2}^{k}\right| .
\end{aligned}
$$

For $\sigma_{1}, \sigma_{2}$ are imaginary and $x_{20} a_{1}<0$, write the last inequality in the form

$$
\begin{aligned}
\left|\tilde{x}_{2 k}\right| \leqslant & \left|x_{10}^{k} x_{20} a_{2}^{k}\right| \int_{0}^{t} \cdots \int_{0}^{s_{2}} \exp \left(\left(k_{1}-\frac{\sigma_{1}^{2}}{2}\right)\left(s_{1}+\ldots+s_{k}\right)\right) 2^{k}(k+1) ! \\
& \times \exp \left(x_{20} a_{1}(1-\ln (2))\left(s_{1}+\ldots+s_{k}\right) \min \left\{1, \exp \left(\left(k_{2}-\frac{\sigma_{2}^{2}}{2}\right) t\right)\right\}\right) d \bar{s}^{k} .
\end{aligned}
$$

For $C=\min \left\{1, \exp \left(\left(k_{2}-\frac{\sigma_{2}^{2}}{2}\right) t\right)\right\}(1-\ln 2) x_{20} a_{1}+k_{1}-\frac{\sigma_{1}^{2}}{2}<0$, after evaluating the integrals we get

$$
\left|\tilde{x}_{2 k}\right| \leqslant\left|x_{10}^{k} x_{20} a_{2}^{k}\right| 2^{k}(k+1) ! \frac{1}{k !|C|^{k}} .
$$

If $\delta=\left|\frac{2 x_{10} a_{2}}{C}\right|<1 \quad \tilde{x}_{2}(t)$ has a limiting value as the dimension of the truncated system $n$ tends to infinity and we suppose that this limiting value is the solution of infinite system. The estimate for the error of the transition from the infinite to the truncated system is $\left|x_{20}\right| \sum_{j=n}^{\infty}(j+1) \delta^{j} \leqslant 2\left|x_{20}\right| n \frac{\delta^{n}}{1-\delta}$.

\section{Example}

Let us evaluate the mathematical expectation from the solutions $\tilde{x}_{1}, \tilde{x}_{2}$ for small values of $a_{1}, a_{2}$. In this case, we can use only the first terms in the expansion with respect to the degrees of $a_{1}, a_{2}$. For example, taking into account the zero and first degrees of $a_{1}, a_{2}$, one infers that

$$
\begin{aligned}
& M \tilde{x}_{1} \approx x_{10} \exp \left(k_{1} t\right)\left(1+x_{20} a_{1} \frac{\exp \left(\left(k_{2}+\sigma_{1} \sigma_{2}\right) t\right)-1}{k_{2}+\sigma_{1} \sigma_{2}}\right), \\
& M \tilde{x}_{2} \approx x_{20} \exp \left(k_{2} t\right)\left(1+x_{10} a_{2} \frac{\exp \left(\left(k_{1}+\sigma_{1} \sigma_{2}\right) t\right)-1}{k_{1}+\sigma_{1} \sigma_{2}}\right) .
\end{aligned}
$$

\section{References}

[1] M. Arato, A famous nonlinear stochastic equation (Lotka-Volterra model with diffusion), Hung. Appl. Math. Comp. Appl. Math. Comput. Modelling, 38 (2003), no. 7-9, pp. 709-726.

[2] N. N. Bogolyubov and D. V. Shirkov, Introduction to Theory of Quantum Fields, Nauka, Moscow, 1976. 
[3] L. Galfi and Z. Racz, Properties of the reaction $A+B \rightarrow C$ type reaction-diffusion process, Phys. Rev. A, 38 (1988), p. 3151.

[4] I. I. Gikhman and A. V. Skorokhod, Stochastic Differential Equation, Naukova dumka, Kiev, 1968.

[5] B. Heidel, C. M. Knobler, R. Hilfer, and R. Bruinsma, Pattern formation at liquid interfaces, Phys. Rev. Lett., 60 (1988), p. 2492.

[6] V. I. Klyatskin, Stochastic Equation and Waves in Random-Inhomogeneous Medium, Nauka, Moscow, 1980.

[7] K. Kowalski and W.-H. Steeb, Nonlinear Dynamical Systems and Carleman Linearization, World Scientific Publishing, Singapore, 1991.

[8] D. F. Kuznetsov, Numerical Integration of Stochastic Differential Equation, S.-Peterburg, 2001.

[9] H. Larralde, M. Araujo, S. Havlin, and H. E. Stanley, Reaction front for $A+B \rightarrow C$ diffusion-reaction systems with initially separated reactants, Phys. Rev. A, 46 (1992), pp. 855-859.

[10] V. P. Maslov, Complex Markov Chains and Continual Feynman Integral, Nauka, Moscow, 1976.

[11] F. J. Muzzio and J. M. Ottino, Evolution of a lamellar system with diffusion and reaction: A scaling approach, Phys. Rev. Lett., 63 (1989), p. 47.

[12] T. Witten and L. M. Sander, Diffusion-limited aggregation, a kinetic critical phenomenon, Phys. Rev. Lett., 47 (1981), p. 1400. 